\title{
Cluster Chemical Ionization and Deuterium Exchange Mass Spectrometry in Supersonic Molecular Beams
}

\author{
Shai Dagan and Aviv Amirav \\ School of Chemistry, Sackler Faculty of Exact Sciences, Tel Aviv University, \\ Ramat Aviv 69978, Tel Aviv, Israel
}

\begin{abstract}
A cluster-based chemical ionization method has been developed that produces protonated molecular ions from molecules introduced through a supersonic molecular beam interface. Mixed clusters of the analyte and a clustering agent (water or methanol) are produced in the expansion region of the beam, and are subsequently ionized by "fly through" electron impact (EI) ionization, which results in a mass spectrum that is a combination of protonated molecular ion peaks together with the conventional EI fragmentation pattern. The technique is presented and discussed as a tool complementary to electron impact ionization in supersonic molecular beams. Surface-induced dissociation on a rhenium oxide surface is also applied to simplify the mass spectra of clusters and reveal the analyte spectrum. The high gas flow rates involved with the supersonic molecular beam interface that enable the easy introduction of the clustering agents also have been used to introduce deuterating agents. An easy-to-use, fast, and routine on-line deuterium exchange method was developed to exchange active hydrogens $(\mathrm{NH}, \mathrm{OH})$. This method, combined with electron impact ionization, is demonstrated and discussed in terms of the unique information available through the EI fragmentation patterns, its ability to help in isomer identification, and possible applications with fast gas chromatography-mass spectrometry in supersonic molecular beams. (J Am Soc Mass Spectrom 1996, 7, 550-558)
\end{abstract}

A mong the molecules that can be ionized by electron impact ionization (EI), there is a significant portion that suffers extensive fragmentation during the ionization process and therefore presents less informative mass spectra, because the molecular ion peak is absent. In these cases, chemical ionization (CI) usually is employed, and it provides a much softer ionization process that allows the formation of a protonated molecular ion peak (which is often the only peak in the spectrum). Positive ion CI is, in practice, less efficient than EI and is selective against molecules that have low proton affinity. The operation of $\mathrm{CI}$ usually requires a change of ion source (except for some ion trap instruments) and involves frequent maintenance and cleaning of the high pressure $\mathrm{CI}$ ion source $[1,2]$.

When a supersonic molecular beam (SMB) is interfaced with an EI ion source [3-5], the intramolecular vibrational cooling effect at the expansion region of the $\mathrm{SMB}$ allows much softer ionization than conventional EI. Electron impact ionization in SMB is characterized by mass spectra that feature an enhanced molecular ion peak combined with the conventional EI frag-

Address reprint requests to Professor Aviv Amirav, School of Chemistry, Sackler Faculty of Exact Sciences, Tel Aviv University, Ramat Aviv 69978, Tel Aviv, Israel. mentation pattern $[3,6,7]$. However, among the subset of volatile molecules that are amenable for $\mathrm{EI}$, there are still a few molecules in which even EI in SMB is not sufficiently soft and the molecular ion peak either does not appear or is still relatively small. Based on the phenomenon of cluster formation in the expansion region of SMB $[8,9]$, we describe here a complementary ionization method called cluster chemical ionization, in which a mixed cluster of the analyte and a clustering agent in the SMB is produced, followed by EI ionizalion and cluster desolvation. This method was developed to produce softer ionization, analytically available to the few molecules that lack molecular weight mass spectral information even with EI in SMB.

An interesting application that has been addressed with conventional $\mathrm{CI}$ is on-line deuterium exchange of active hydrogens to obtain more information on the molecular structure as well as to assist in resolution of complex mass spectrometry and gas chromatography-mass spectrometry (GC-MS) data. First demonstrated and studied by Hunt et al. [10], this technique now has several applications [11-14], which include the successful applications by Verma et al. to fast-atom bombardment ionization [15] and by Siegel to thermospray ionization [16-17]. With CI, deuterium exchange ratios are not necessarily high due to reverse $\mathrm{D} / \mathrm{H}$ exchange, and the system must be treated care- 
fully (deuterium surface conditioning) to avoid interference from water (that always exists to a certain degree in the gas lines, vacuum chamber, and CI ion source). Due to the nature of CI, extra information usually can be gained only for the molecular ion peak, by its mass shift. With SMB interface, deuterium exchange becomes easy to apply and produces unique spectra that will be presented and discussed in this article.

Preliminary results of this work reported by us [18] coincided with a separate work by Wang and Evans [19], who investigated similar issues. These two studies were carried out in an independent manner and were aimed at different applications with different molecular systems.

\section{Experimental}

The experimental apparatus is described in detail elsewhere $[7,20]$. Briefly, a SMB interface, which includes a continuous flow nozzle and a differentially pumped vacuum system, allows the introduction of up to 240 $\mathrm{mL} / \mathrm{min}$ of $\mathrm{He}$ or $\mathrm{H}_{2}$ carrier gas. Sample molecules are introduced into the gas stream by means of a lab-made direct sample insertion device or by gas chromatography (GC) injection, into a 50-cm megabore column. A conventional gas chromatograph (Varian 3400, Varian Associates, Walnut Creek, CA) also is coupled to the SMB interface. It is equipped with short columns and usually is operated at high gas flow rates to allow different fast GC time scales to be obtained [7]. After expansion from the nozzle and collimation, the sample molecules enter the ionization and detection chamber. Electron-impact ionization is achieved by a home-made "fly through" open Brink-type ion source [21] that is operated in a mode of electrostatic "background ion filtration" $[3,5,6]$, where only bearm-cooled molecules (that have kinetic energies of typically 1-10 eV with $\mathrm{He}$ carrier) pass through a blocking potential, are distinguished from thermal background molecules, and are analyzed. Throughout this work, 70-eV electron energy was used, although the electron energy could be controlled to reduce the degree of ion fragmentation [7]. After ionization, ions are deflected by $90^{\circ}$ (utilizing an electrostatic mirror ion deflector mounted on a XYZR manipulator) and are analyzed by a quadrupole mass analyzer (VG-SXP600, VG Quadrupole Ltd., Manchester, UK). Changes in the manipulator position allow a rhenium oxide surface to replace the ion deflector in front of the beam line and to serve for surface-induced dissociation experiments [22] as well as for hyperthermal surface ionization $[4,20,23]$ (not used in the current report).

Clusters are produced by introduction of reagent gases (vaporized liquids) into the gas stream through a temperature-controlled direct insertion device or through a flow-controlled make-up gas line (connected between the end of the $50-\mathrm{cm}$ column and the nozzle).
Because the gas line is not heated, it was used only with the relatively volatile methanol. Reagent gases were introduced into the He carrier gas with typical liquid flow rates of $\sim 5-12 \mu \mathrm{L} / \mathrm{min}$, up to $\sim 20$ $\mu \mathrm{L} / \mathrm{min}$. In the deuterium exchange experiments, $\mathrm{D}_{2} \mathrm{O}$ or $\mathrm{CD}_{3} \mathrm{OD}$ was introduced only through the heated insertion device to prevent interference from water adsorbed on cold metal surfaces.

All the cluster CI experiments were performed with a capillary-heated Vespel $^{\mathrm{R}}$ nozzle (homemade) constructed of a 15-mm-long, 3-mm-diameter Vespel rod with a 1-mm diameter inner hole. The nozzle hole was drilled $0.7 \mathrm{~mm}$ from the vacuum outer side and had a diameter of $100 \mu \mathrm{m}$. The nozzle was heated to $\sim 200$ ${ }^{\circ} \mathrm{C}$. This nozzle exhibited better cluster formation efficiency than a $150-\mu \mathrm{m}$-diameter 5 -mm-long fused silica capillary tube that also was tested. Cluster formation can be further enhanced with conical nozzles and higher backing pressures. However, consideration of the cooling efficiency (important to EI-SMB) and forward beam flux made us decide to use a "medium length" capillary nozzle operated at the convenient pressure of $1 \mathrm{~atm}$, which allows atmospheric pressure sampling. Thus, this nozzle is the same as that used in EI-SMB mass spectrometry and is operated at the same pressures, flows, and temperatures. Helium served as a carrier gas for all the measurements unless otherwise indicated. Argon, which is a better clustering carrier, is far inferior to helium in terms of forward flux (i.e., the efficiency of jet separation and molecular flux at the ion source) and therefore was not preferred. We examined several clustering agents, which included water, methanol, ethanol, cyclohexane, pentane, acetoniltrile, and acetone, and found that water and methanol exhibited the best results in terms of efficiency of protonated molecular ion peak formation, whereas methanol was the easiest to use and formed fewer self-clusters than water.

\section{Results and Discussion}

\section{Cluster Chemical Ionization}

Reviewing a large number of molecules analyzed by EI-SMB that included straight chain and branched alkanes [7], carbamate pesticides, steroids, and drugs [24], chlorinated pesticides and others, we observed that the vast majority of these molecules exhibit a significant molecular ion peak that in many cases does not appear with conventional 70-eV EI. However, there are some rare cases in which this does not occur. One such case is aldicarb sulfone. This thermally labile carbamate pesticide suffers also from extensive ion dissociation in EI. Figure 1a shows the NIST library spectrum of aldicarb sulfone, which features only fragment ions (the molecular mass is 222). In the absence of the molecular ion peak, several different carbamates (which include aldicarb sulfone) and their decomposi- 


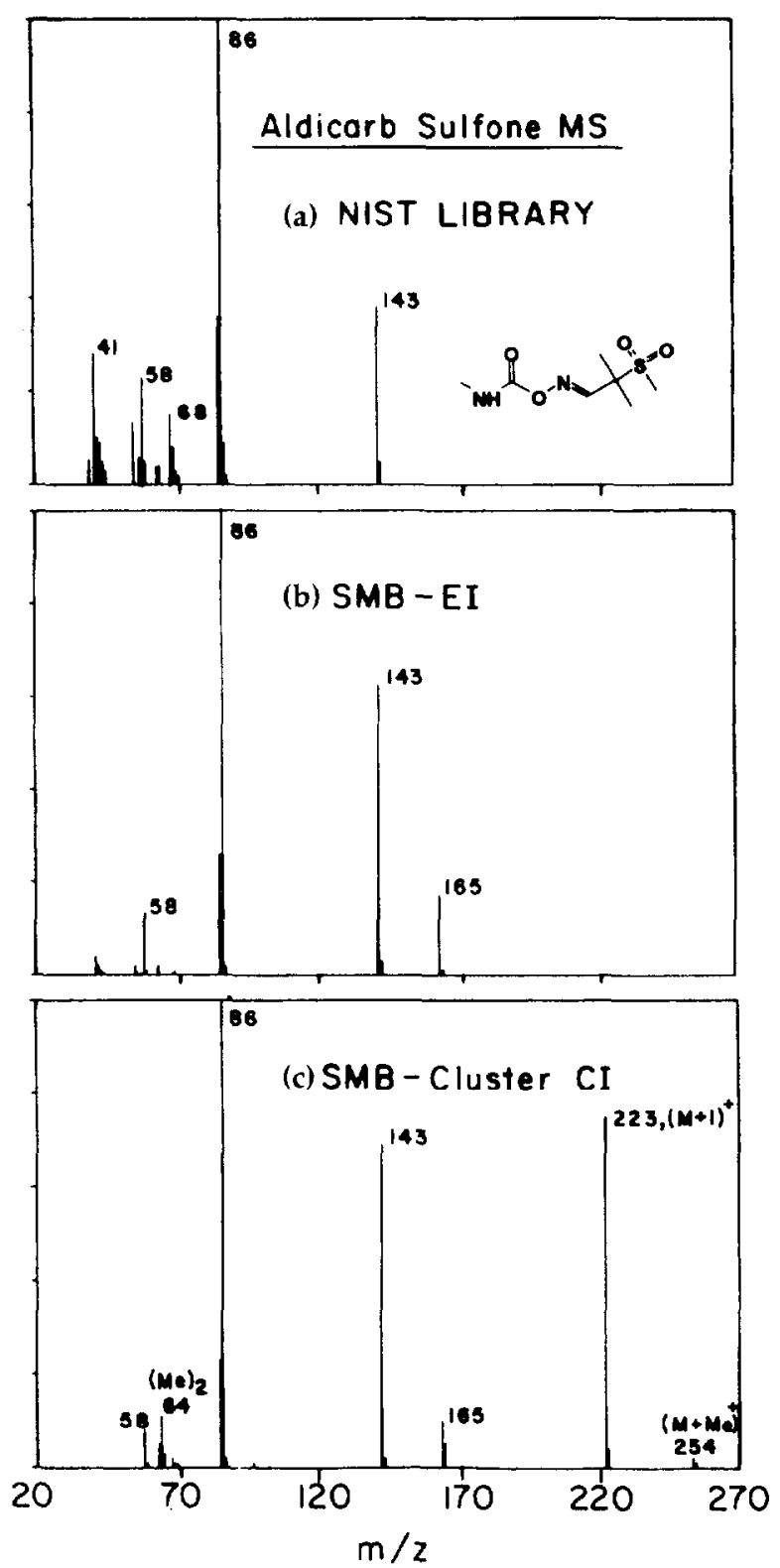

Figure 1. Mass spectra of aldicarb sulfone in SMB. EI in SMB (b) is softer and more informative than the NIST spectrum (a), but molecular peak information is obtained only through cluster $\mathrm{CI}$ (c). Clusters were formed with methanol, and the $[\mathrm{M}+\mathrm{Me}]^{+}$ peak is the cluster ion of aldicarb sulfone with one methanol molecule. Fast GC-MS was performed to achieve the spectra in (b) and (c), where the $50-\mathrm{cm}$ column was heated to $140^{\circ} \mathrm{C}$.

tion products show similar mass spectra. In Figure $1 \mathrm{~b}$, we present the EI-SMB mass spectrum of aldicarb sulfone (obtained by fast GC-MS [24]), which features the new unique $m / z 165$ fragment ion and enhanced $\mathrm{m} / z 143$ peak; still the molecular peak is not observed. Figure 1c demonstrates the mass spectrum obtained when methanol is introduced into the He gas stream. A predominant $[\mathrm{M}+1]^{+}$peak is now added to the EI fragments, together with a small $m / z 254$ peak (a cluster ion of the analyte and one methanol molecule) and a small neighboring protonated cluster ion peak $(m / z 255)$. A protonated fragment peak also is observed at $m / z$ 166. Self-clustered methanol can be observed at the dimer peaks around $m / z$ 64. (Actually the methanol dimer peaks appeared at $m / z 63,64$, and 65 with intensity ratios that depended upon the methanol flux. At lower flux the $m / z 63$ peak was dominant, whereas at high methanol flux the $m / z 65$ became dominant.) 'Ihis overall combination of $\mathrm{EL}$ and $\mathrm{CI}$ information has been achieved with EI sensitivity (EI-SMB sensitivity is comparable to conventional EI [7]).

Another example of a molecule that does not exhibit a molecular ion peak in conventional EI is dioctylphthalate (DOP) (we have measured $10^{-6}$ relative abundance in a conventional magnetic sector instrument). In FI-SMB, DOP exhihits a small $(\sim 5 \%)$ molectilar ion peak at $m / z$ 390, as seen in Figure 2a. The mass spectrum of methanol-clustered DOP is portrayed in Figure $2 \mathrm{~b}$, where a prominent protonated molecular ion peak at $m / z 391$ is shown together with the DOP(methanol) cluster peak at $m / z$ 422. This mass spectrum was obtained with moderate amounts of methanol ( $\sim 12-15-\mu \mathrm{L}$ liquid per minute) that caused only minor reduction in the total intensity. We found that increased amounts of clustering agent allowed larger clusters of $\mathrm{DOP}$ (methanol) ${ }_{1 \text {, }}$ to be observed with more dominant protonated molecular peaks, but the overall intensity was reduced. This reduction probably is duc to less efficient jet focusing caused by increased amounts of solvent in the expanding jet.

We have applied cluster $\mathrm{CI}$ to several other molecules. Aldicarb, nicotine, lidocaine, and phentermine also presented a $[\mathrm{M}+1]^{+}$peak, sometimes with some enhancement in the molecular peak itself (which is originally small). On the other hand, no effect was observed with aromatic compounds (such as anthracene) or with straight and branched chain alkanes (such as hexadecane or squalane). Fortunately, aromatics exhibit a dominant molecular peak even with conventional $\mathrm{EI}$, and the alkanes, which do sutfer from extensive fragmentation with conventional EI, exhibit predominant molecular ion peaks with EI-SMB [7]. As a general rule, as in conventional $\mathrm{CI}$, molecules with relatively high proton affinity are more likely to be protonated in the cluster $\mathrm{CI}$ process. In all the effective cases mentioned, this soft ionization was characterized by the appearance of the EI fragmentation pattern together with the protonated molecular ion peak. Therefore, cluster $\mathrm{CI}$ produces an overall softer ionization than EI-SMB, but is still not as soft as conventional $\mathrm{CI}$ in which in many cases the protonated molecular peak appears alone in the spectrum. We note also that cluster CI in SMB is easy to switch to and its operation can even be automated. This automation can be achieved by opening a solenoid valve outside the vacuum system. Cluster CI also is easy to apply because the clustering agents are liquids rather than gases (no gas cylinders and pressure regulators are needed). 


\section{Dioctylphtholate}
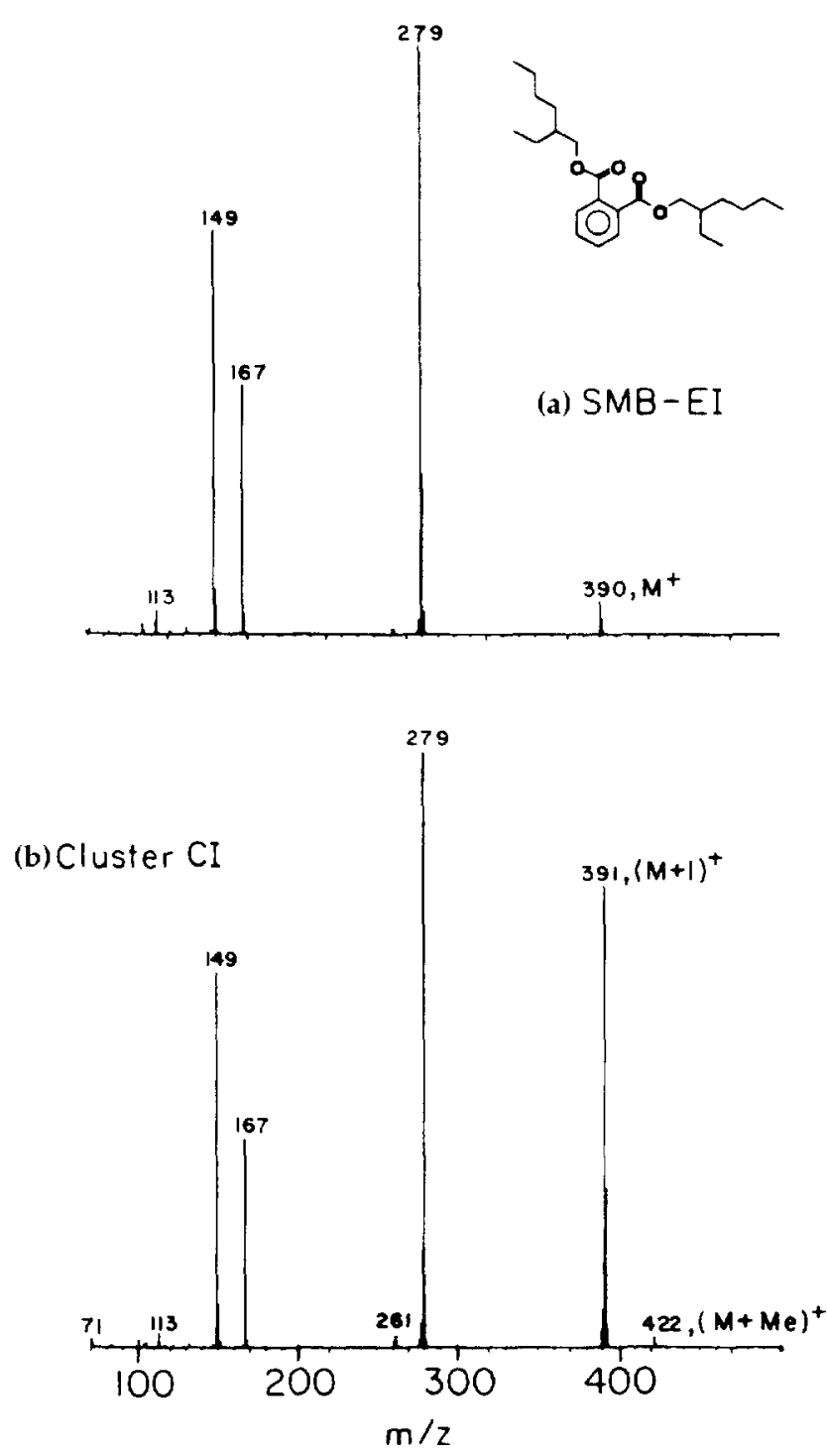

Figure 2. Mass spectra of dioctylphthalate (DOP) in SMB. The molecular ion peak information in EI with SMB (a) is enhanced by cluster $\mathrm{CI}$ (b). Clusters were formed with methanol that was introduced at a liquid flow rate of $\sim 15 \mu \mathrm{L} / \mathrm{min}$. The $[\mathrm{M}+\mathrm{Me}]^{+}$ peak is the cluster ion of DOP with one methanol molecule.

Finally, cluster CI is characterized by sensitivity similar to that of EI in SMB [7], and the overall ionization yield is uniform like in EI-the result of a small effect of the solvent molecules on both the analyte flux and its ionization cross section. In cases where single ion monitoring (SIM) is applied to the protonated $[\mathrm{M}+1]^{+}$ peak, a practical increase in sensitivity can be achieved.

The mechanism of cluster CI is not clear yet, but based on existing data we suggest two possible models: 1) A simple model based on the assumption that the clustering efficiency is not high and only a portion of the molecules produce clusters. Thus a bimodal distribution exists, where clustered molecules produce protonated molecular ions (through intracluster hydro- gen atom transfer as in the classical CI reagent gas reaction $\left.\mathrm{CH}_{4}^{+}+\mathrm{CH}_{4} \rightarrow \mathrm{CH}_{5}^{+}+\mathrm{CH}_{3}\right)$, followed by cluster desolvation that pumps out vibrational excess energy and minimizes fragmentation. Coexisting unclustered molecules produce the standard EI-SMB fragmentation pattern that is superimposed on the overall mass spectra obtained. It is more likely, however, that most of the analyte molecules form clusters whose sizes are much larger than those observed in the EI mass spectra. In such a case, another model has to be addressed: 2) A model in which two possible ionization processes can occur in the cluster. The first is a collision of the ionizing electron with the analyte itself and the second is the collision of the ionizing electron with one of the clustering agents attached to the analyte. In the first case, after the EI ionization process and while the cluster is fully or partially desolvated, a hydrogen atom attaches to the molecular ion from the cluster to form a protonated molecular ion that "pumps out" the distributed excess vibrational energy gained in the system by the ionization process. In this case, soft ionization can be achieved. In the second case, when ionization is at the clustering agent, electron transfer from the analyte to the cluster also is involved and is favored by the lower ionization potential of the analyte molecule. In addition to the EI induced vibrational energy, this process entails additional excess energy due to differences between the high ionization potential of the clustering agent and the relatively low ionization potential of the analyte molecule (presumably a difference of more than $3 \mathrm{eV}$ ). This excess energy can promote ion dissociation and form the fragmentation pattern that is always present with the cluster CI spectra. At this time, based on existing data, we cannot determine the practical weight of each of the mechanisms suggested.

\section{Surface-Induced Dissociation of Clustered Molecules}

The size of clusters that can be formed during the free jet expansion depends upon, among other things, the amount of clustering agent introduced. In cases where large amounts of clustering agent are introduced, two kinds of interfering clusters can dominate the mass spectrum. One type of interfering clusters is self-clusters of the clustering agent that are easily produced. Water, for example, very easily forms selfclusters of ionized $\left(\mathrm{H}_{2} \mathrm{O}\right)_{n}$ (with or without additional hydrogen) that can cover the lower range of the mass spectrum after ionization. The other type of interfering clusters is excess clustering of the analyte with clustering agents $\left[\mathrm{M}\left(\mathrm{H}_{2} \mathrm{O}\right)_{n}\right.$ for instance] that can cover the spectral range from the molecular ion peak onward. This effect increases with easily clustered sample compounds. Both cases are analytically undesirable, because the mass spectrum information of the analyte becomes difficult to extract from the complex mass spectrum picture. 
This difficulty may be overcome by gas-phase collision-induced dissociation (CID) that can strip clusters to expose the bare analyte molecule [19]. Another alternative is surface-induced dissociation (SID), which is a developing competing technique [25-28]. We used SID on a rhenium oxide surface [22] to simplify the complex picture achieved under heavily clustered sampling. In Figure 3, nicotine was analyzed in the presence of high quantities (more than $10-\mu \mathrm{L} / \mathrm{min}$ liquid flow rate) of methanol. The EI-SMB spectrum of the bare molecule (Figure 3a) was buried under selfclusters of protonated (methanol) 1 at masses 65, 97, and 129 (Figure $3 \mathrm{~b}$ ). Note also the protonation effect on the nicotine molecule itself at $m / z 163[\mathrm{M}+1]$. Following low energy $(\sim 8-\mathrm{eV})$ collision of the inns presented in Figure $3 b$ with the $\mathrm{ReO}_{2}$ surface, the methanol clusters decreased significantly and the nicotine spectrum was exposed (Figure 3c). The $m / z 161$ peak $[\mathrm{M}-1]^{+}$is a typical peak for low energy SID of nicotine on $\mathrm{ReO}_{2}$ (as well as for hyperthermal surface ionization of this molecule [23]). Other peaks are identical to the EI peaks.
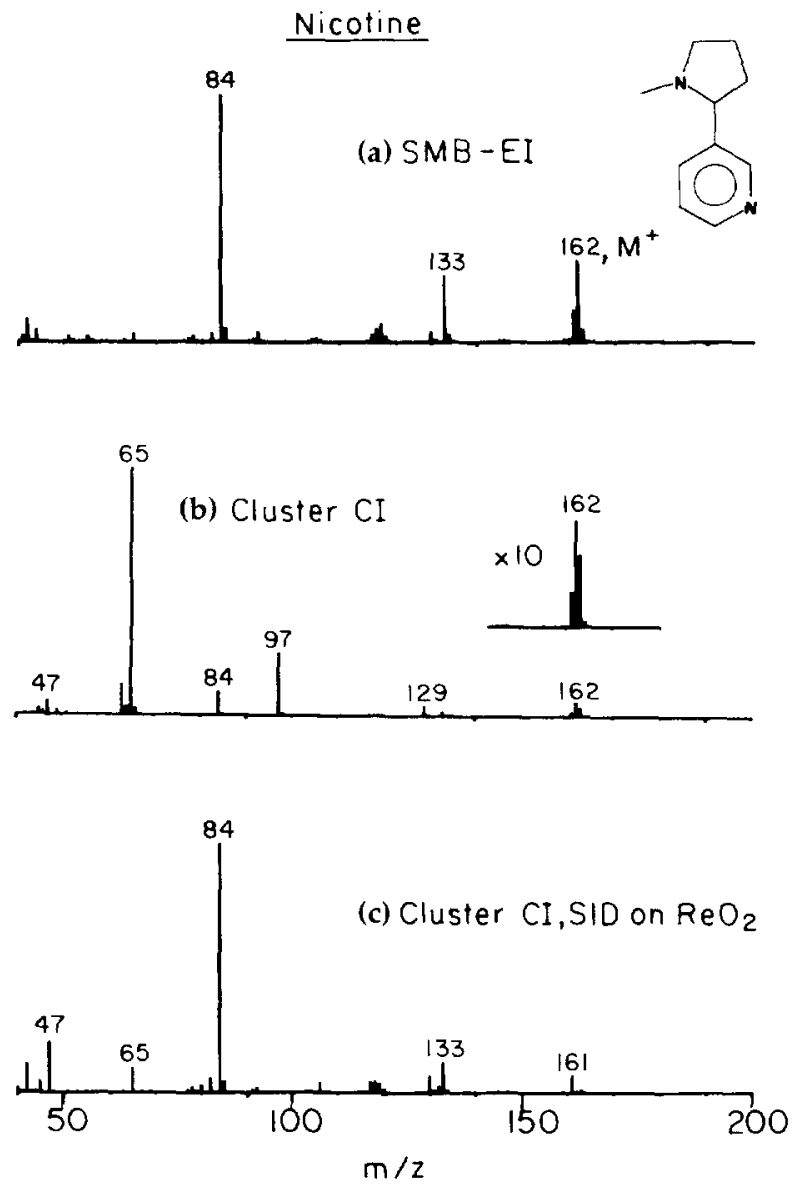

Figure 3. Mass spectra of nicotine in the three indicated modes: a spectrum with enhanced molecular ion peak in EI-SMB (a); a protonated molecule spectrum achieved with cluster $\mathrm{CI}$ with interference from dominant self-clusters of methanol (b); and the ion outcome of cluster stripping by surface-induced dissociation on a rhenium oxide surface at $\sim 8-\mathrm{eV}$ collision energy (c).
A more complex example is heavily clustered DOP. For this purpose we clustered DOP with water in a carrier gas mixture of argon and helium. Argon was added to the carrier gas to enhance the efficiency of cluster formation. In Figure $4 a$ the resulting spectrum is presented. The DOP spectrum is partially hampered by self-clusters of water (the lower mass peaks with $m / z \quad 18$ spacing) and higher mass clusters of $\mathrm{DOP}\left(\mathrm{H}_{2} \mathrm{O}\right)_{11}$ at $m / z 408$ and higher. Because DOP is a very fragile molecule, it was impossible to strip $\mathrm{H}_{2} \mathrm{O}$ off the clusters without causing complete dissociation of the DOP itself. However, low energy SID on the $\mathrm{ReO}_{2}$ surface produced the spectrum shown in Figure $4 \mathrm{~b}$ that is dominated by the $m / z 149$ characteristic phthalate fragment, which is also the dominant peak in the EI library spectrum of DOP (Figure $4 c$ ).

The successful exposure of the characteristic molecular fragment peaks from the cluster background is explained by the fact that the weakly bound clusters have a much lower threshold for surface-induced dissociation than the organic molecule. Furthermore, because the ionization potential of the self-clusters, and
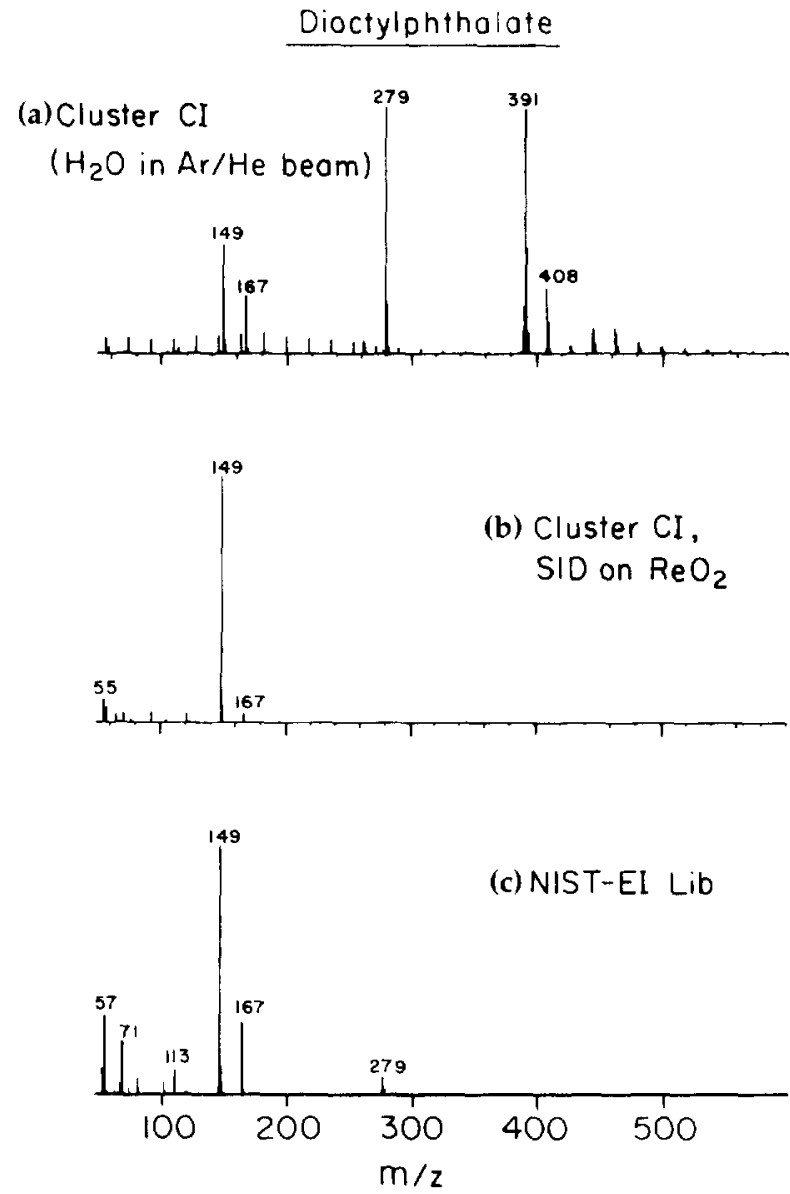

Figure 4. Mass spectra of dioctylphthalate (DOP) in the three indicated modes: $\mathrm{He} / \mathrm{Ar}$ carrier gas $(\sim 20 \%$ argon), which formed molecular clusters and water self-clusters (a); cluster stripping by surface-induced dissociation on a rhenium oxide surface at $\sim 8 \mathrm{eV}$ collision energy, which exposed the dominant EI fragments of DOP (b); comparison with the NIST spectrum (c). 
especially of the cluster agents alone [10.84 eV for methanol (CRC handbook)], is assumed to be higher than the typical ionization potential of the analyzed molecule, their neutralization probabilities near the surface are much higher. Therefore mostly ions related to analyte molecules are observed in the SID mass spectrum picture.

\section{Deuterium Exchange Labeling}

The SMB sample interface is very inert (because it is designed as a GC interface) and is operated with very high gas flow rates $(240 \mathrm{~mL} / \mathrm{min}$ through the nozzle). These features make it easy to obtain efficient deuterium exchange reactions for labile hydrogens $(\mathrm{OH}$, $\mathrm{NH}$, and possibly SH groups). A 17- $\beta$-estradiol EI-SMB mass spectrum is shown in Figure $5 a$ and is compared to its deuterium-exchanged EI-SMB mass spectrum in Figure $5 \mathrm{~b}$. Because $\beta$-estradiol has two $\mathrm{OH}$ locations with active hydrogens, a mass shift of two mass units from the original $\mathrm{M}^{+}$peak at $m / z 272$ indicates that the two $\mathrm{OH}$ sites have been exchanged. Note that this molecule does not exhibit any protonation effect at these reagent levels of seeding, so that all mass shifts stem only from deuteration. From the relative abundance of the 272 peak in the expanded part of Figure $5 \mathrm{~b}$ minus the natural abundance of the 270 peak in Figure $5 \mathrm{a}$, it is estimated that less than $1 \%$ of the molecules did not undergo hydrogen exchange at all. By comparing the 273 peak in Figure $5 b$ to the 271 peak in Figure $5 \mathrm{a}$ in the same way, we also estimate that in less than $5 \%$ of the molecules only one hydrogen was exchanged. The spectra in Figure $5 a$ and $b$ differ not only in the molecular peak, but also exhibit mass shifts in the fragment ions. The $m / z 133,146$, 160,170 , and 213 ions in Figure $5 \mathrm{a}$ are shifted by one mass unit, whereas the $m / z 186$ ion peak is shifted by two mass units to $m / z 188$. This unique structural information that is gained from the fragmentation pattern and that provides the number of $\mathrm{OH}$ sites in each fragment is not available in CI.

Lidocaine is another example of a molecule that contains an active hydrogen (one $\mathrm{NH}$ group, labile to deuterium exchange). This molecule has a high proton affinity, so it also accepts a proton in cluster CI. In Figure 6 these combined phenomena are portrayed. Figure 6a shows the EI-SMB of lidocaine (the molecular peak at $m / z 234$ is increased compared to the NIST library, where it is only a few percent). Cluster CI with methanol (Figure 6b) produces the protonated molecular peak at $m / z$ 235, and exposure to deuterated methanol forms the combination shown in Figure $6 \mathrm{c}$. The $m / z 235$ peak $[\mathrm{M}+1]$ is the $\mathrm{H} / \mathrm{D}$ exchanged molecular peak, where the $m / z 237$ peak [M + 3] represents exchanged molecules that are also cluslered by a deuterating agent. The peak at $m / z 236$ is a natural $[M+1]$ peak of the deuterated moleculc, possibly with the minor addition of dcuterium clustered-unexchanged molecules. The peak at $m / z$

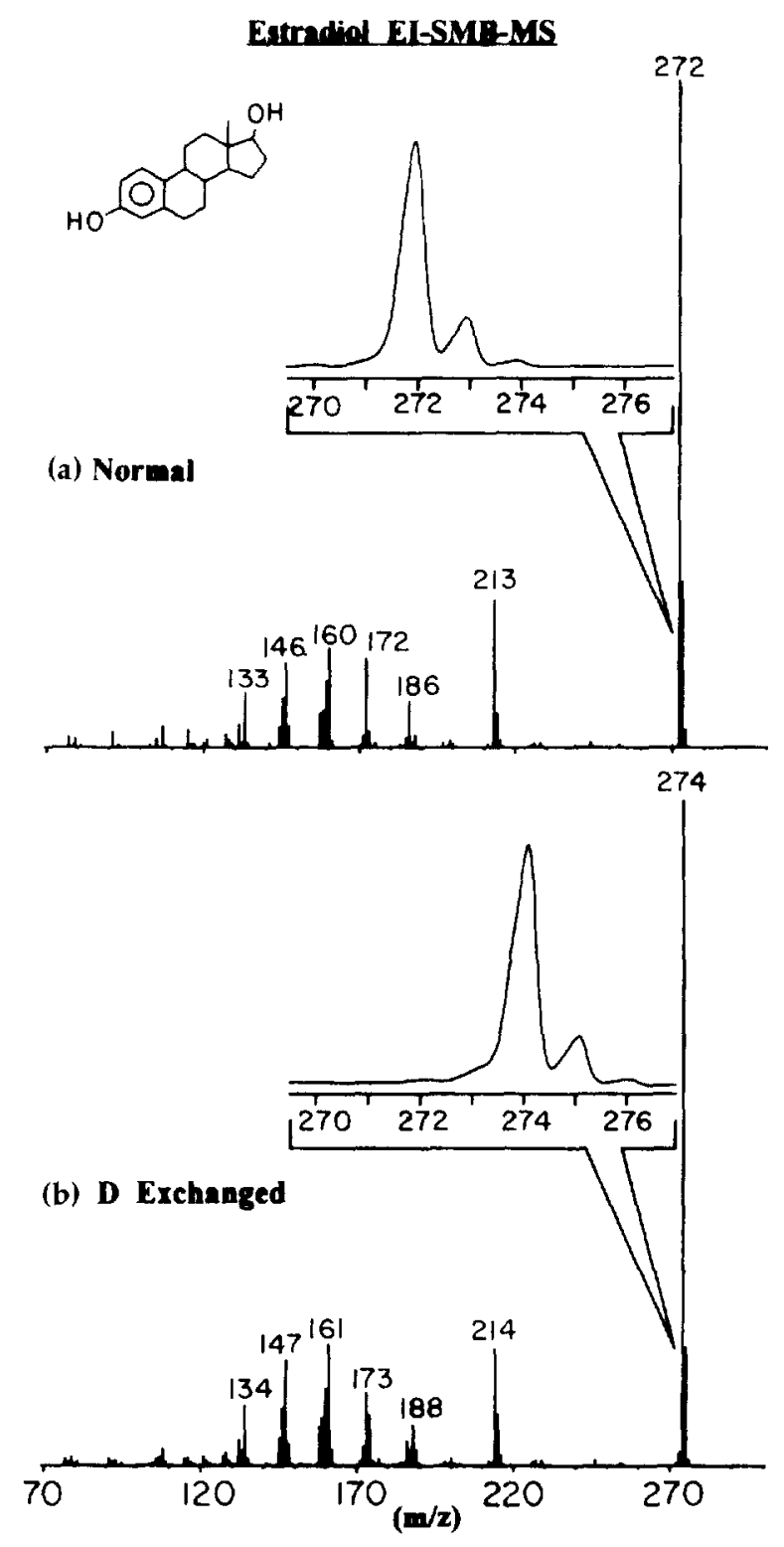

Figure 5. Deuterium exchange of hydrogens in alcohol $(\mathrm{OH})$ groups. EI mass spectra of $\beta$-estradiol in the normal (a) and deuterated (b) modes are shown. Mass shifts are observed in the molecular ion peak as well as in the fragment ions. $D_{2} \mathrm{O}$ was used at a liquid flow rate of $\sim 7 \mu \mathrm{L} / \mathrm{min}$.

234 is probably due to incomplete exchange. Like $\beta$ estradiol, structural information on lidocaine also can be elucidated from the fragmentation pattern, where it is clear that only the $m / z 120$ fragment (exchanged to 121) carries the active hydrogen.

The kinetics of switching to and from the deuterium exchange mode also were explored. A vial that contained $\mathrm{D}_{2} \mathrm{O}$ was alternately inserted into the system for $10 \mathrm{~s}$ and taken out for $20 \mathrm{~s}$. A kinetics graph (Figure 7) was obtained by monitoring the $m / z 272$ and 274 peaks of $\beta$-estradiol that was continuously introduced from the gas chromatograph by a home-made direct sample introduction device (mounted on the gas chromatograph injector [patent pending]). The time for full 

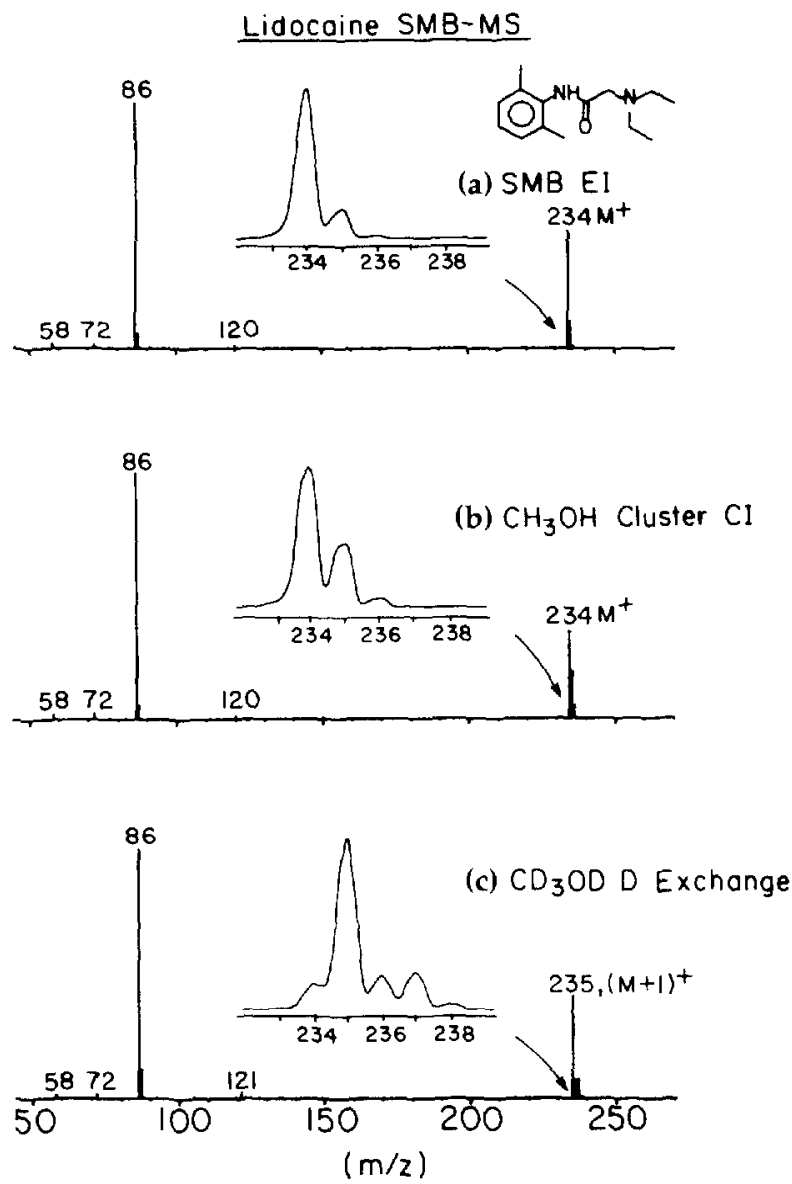

Figure 6. Deuterium exchange of hydrogens in amine (NH) groups. Mass spectra of lidocaine are shown in the three indicated modes: enhanced molecular peak in EI-SMB (a); some protonation in cluster $\mathrm{CI}$ with methanol (b); combined deuterium exchange and protonation with deuterated methanol (c).

deuteration $(\mathrm{H} / \mathrm{D})$ is estimated to be less than $3 \mathrm{~s}$, whereas the natural recovery $(\mathrm{D} / \mathrm{H})$ time is about $20 \mathrm{~s}$. The kinetics became much slower (minutes) with lower helium gas flow through the line and nozzle (less than $240 \mathrm{~mL} / \mathrm{min}$ ). We also performed this kinetics experiment by alternately replacing a $\mathrm{D}_{2} \mathrm{O}$ vial with an $\mathrm{H}_{2} \mathrm{O}$ vial. Here the periods for full $\mathrm{H} / \mathrm{D}$ and $\mathrm{D} / \mathrm{H}$ exchange were the same, about $2-3 \mathrm{~s}$. Introduction of $\mathrm{CD}_{3} \mathrm{OD}$ vapor into the gas stream in the unheated stainless steel (SS) gas line of the make-up gas connection limited the exchange efficiency to only $\sim 20 \%$ with very slow kinetics. This decreased efficiency is probably due to trace amounts of water adsorbed on the SS surfaces. This emphasizes the importance of inertness (minimal memory effects) of the transfer line on the exchange efficiency and kinetics.

The observed fast exchange kinetics, which depend on the carrier gas flow rate, pressure, and transfer line inertness, strongly suggest that the efficient deuterium exchange process is completed in the high flow, high pressure regions that include the insertion device, the $50-\mathrm{cm}$ column that leads to the nozzle, and the nozzle itself. Because efficient exchange also has been achieved

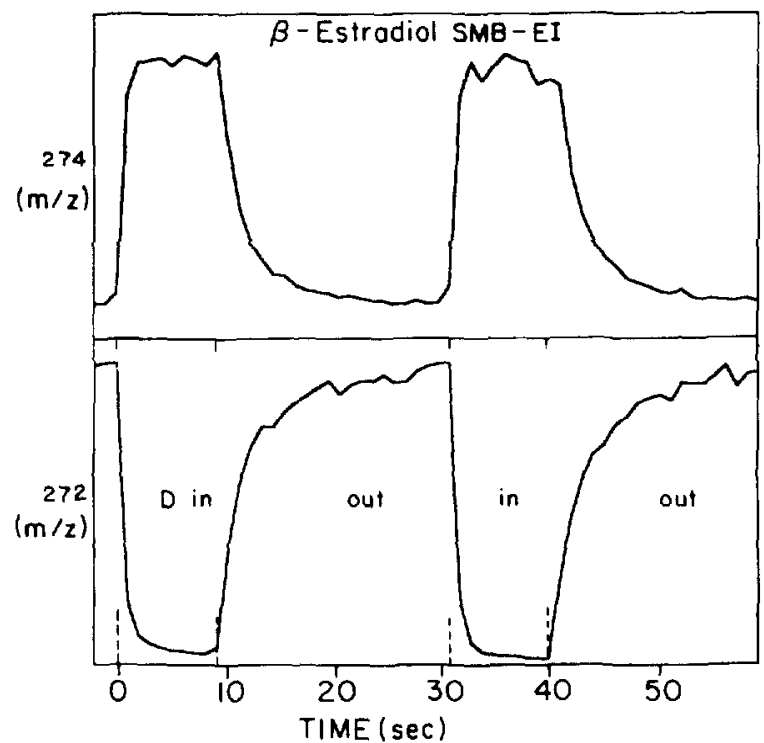

Figure 7. Kinetics of switching to and from the deuterium exchange mode in SMB for $\beta$-estradiol (continuously introduced). A vial that contained $\mathrm{D}_{2} \mathrm{O}$ was alternately and repeatedly inserted for $10 \mathrm{~s}$ and then removed for the next $20 \mathrm{~s}$. The high gas flow rates $(240 \mathrm{~mL} / \mathrm{min})$ and pressures $(760-730$ torr), together with inert surfaces at the inlet and transfer lines, allowed high efficiency of exchange with a very fast response time.

with relatively small quantities of $\mathrm{D}_{2} \mathrm{O}$ or $\mathrm{CD}_{3} \mathrm{OD}$, where cluster $\mathrm{CI}$ is not observed, it is unlikely that the deuterium exchange takes place in the SMB expansion as part of a cluster $\mathrm{Cl}$ process. We also find it less probable that exchange takes place at or after the ion source because the pressure in the ionization region drops to less than $\sim 10^{-3}$ torr and the ion source is based on molecular collision-free "fly through." Deuterium exchange EI-SMB therefore can be used as a routine procedure. It can be obtained within a scale of a few seconds and requires no prior treatment. This procedure has the potential to be automated with a solenoid valve to control the deuterating agent flow.

Apart from the structural information available through deuterium exchange methods, a routine and fast deuterium exchange analysis also can help to resolve some difficulties in distinction between isomers. Two examples are illustrated in Figure 8. Anisole (methoxybenzene) and cresol (3-methylphenol) are isomers that each have a molecular weight of 108 and similar mass spectra. Cresol can undergo deuterium exchange at the $\mathrm{OH}$ group, and this fact can provide an easy and fast distinction between the two isomers. A mixture of the two isomers was injected into a $4-\mathrm{m}$ megabore column in the Varian 3400 gas chromatograph coupled to the SMB system while $\mathrm{D}_{2} \mathrm{O}$ was continuously introduced into the SMB interface to allow $\mathrm{H} / \mathrm{D}$ exchange. The total ion count (TIC) and single ion monitoring (SIM) chromatograms on the left-hand side of Figure 8 describe the results. Both compounds eluted within less than $20 \mathrm{~s}$ with the fast GC. Cresol exchanged one hydrogen and produced an 
D-Exchange Fast GC-EI-SMB-MS

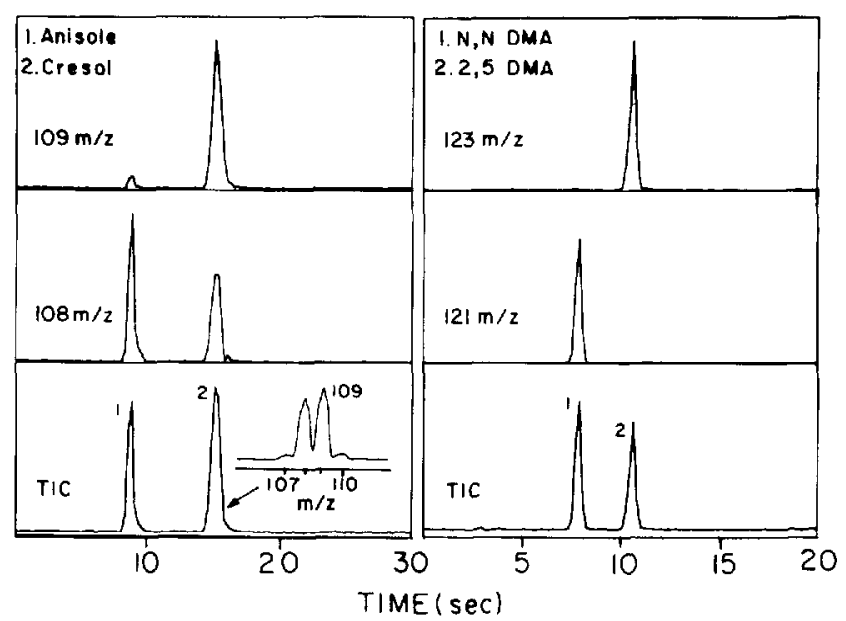

Figure 8. Isomer distinction by deuterium exchange. Fast GCMS of the indicated compounds in the deuterium exchange mode. A 4-m megabore column with a He gas flow rate of $\sim 7$ $\mathrm{mL} / \mathrm{min}$ and isothermal temperature of $75{ }^{\circ} \mathrm{C}$ were used for separation. Deuterating agent $\left(\mathrm{D}_{2} \mathrm{O}\right)$ was introduced continuously into the interface between the gas chromatograph and the nozzle, where a high gas flow rate of $240 \mathrm{~mL} / \mathrm{min}$ exists.

$[\mathrm{M}+1]^{+}$peak at $m / z 109$, shown in the upper trace. Cresol also exhibits an [M -1$]$ peak in its EI spectrum, that becomes a peak at $m / z 108$ after deuterium exchange, as shown in the middle trace as well as in the insert in the lower TIC trace. This peak at $m / z 108$ results from a hydrogen loss from the methyl group on the ring and not from the $\mathrm{OH}$ (OD in this case) group. Anisole, which did not undergo $\mathrm{H} / \mathrm{D}$ exchange, presents the original molecular ion peak at $m / z$ 108. The first small peak that elutes at $m / z 109$ is an original $[\mathrm{M}+1]^{+}$peak of anisole that has a natural abundance of $\sim 8 \%$. Another demonstration is shown on the right-hand side of Figure 8, where two isomers of dimethylaniline (DMA) have molecular weight of 121 . In this case, the N,N-DMA does not have any labile hydrogen, but the 2,5 DMA has two active $\left(\mathrm{NH}_{2}\right)$ sites. A mixture of the two DMA isomers was injected under the same conditions as with the cresol-anisole mixture. The two isomers clearly can be distinguished, as presented in the SIM chromatograms of the original molecular weight mass $(m / z \quad 121)$ and the $[\mathrm{M}+2]$ mass ( $m / z 123$ ). Therefore, in cases where active hydrogen groups exist in the analyte, this easy-to-use technique of deuterium exchange provides an extra dimension of information that also can allow distinction between isomers or between coeluting molecules that have similar mass spectrometry peaks. We believe this labeling and distinction method could have implications especially where fast analysis of target compounds in complex mixtures is needed. In other cases, the sclective protonation in cluster CI potentially can serve for distinction purposes as well.

\section{Conclusions}

A cluster CI technique for soft ionization that is more selective and results in more fragmentation than conventional $\mathrm{CI}$ has been described and discussed. This method, together with EI-SMB, provides CI plus EI information for practically any molecule amenable to EI ionization with EI sensitivity and minimal complexity. For an cxisting SMB system, cluster $\mathrm{CI}$ is a built-in feature and requires little additional cost. Heavily clustered spectra were simplified by SID that caused dissociation and probably selective neutralization of the clustering agents that exposed the bare molecule spectrum. To achieve full stripping of clusters with minimal molecular dissociation, further study is required to seek a surface that features more efficient ion survival probabilities at even lower collision energies. Note, however, that for general analytical applications, extensive cluster formation usually does not occur.

The deuterium exchange method, which is based on the high pressure, high gas flow rates amenable to the SMB interface, produced additional information on $\mathrm{OH}$ and $\mathrm{NH}$ groups very easily and in a fast and routine manner. It also offered extra unique structural information gained from deuterated fragments exhibited through the EI ionization. Although deuterium exchange labeling is not a major technique in analytical mass spectrometry (especially not in the GC-MS field), we feel that with the advantages endowed by $\mathrm{SMB}$, it will be much more attractive, offer a wider range of applications, and add another capability to mass spectrometry and fast GC-MS in SMB.

\section{Acknowledgments}

Funding for this work was provided by grants from the German-Israeli Foundation for Scientific Research and Development, the James Franck Institute for laser matter interactions, and the United States Army through its European Research Office.

\section{References}

1. Chapman, J. R. Practical Organic Mass Spectrometry; Wiley: New York, 1985; Chapters 3 and 4.

2. Harrison, A. G. Chemical Ionization Mass Spectrometry, 2nd ed.; CRC, Boca Raton, FL, 1992.

3. Amirav, A.; Danon, A. Int. J. Mass Spectrom. Ion Processes 1990, 97, 107-113.

4. Amirav, A. Org. Mass Spectrom. 1991, 26, 1-17.

5. Amirav, A.; Danon, A. Mass Spectrometer Method and Apparatus for Analyzing Matcrials; U.S. Patent 5055677, 1991, Israel and European patent applications accepted.

6. Amirav, A. J. Phys. Chem. 1990, 94, 5200-5202.

7. Dagan, S.; Amirav, A. J. Am. Soc. Mass Spectrom. 1995, 6, $120-131$

8. Sievert, R.; Cadez, I.; van Donz, J.; Castelman, A. W., Jr. J. Phys. Chem. 1984, 88, 4502-4505.

9. Jonkman, H.; Even, U.; Kommandeur, J. J. Phys. Chem. 1985, $89,4240-4243$.

10. Hunt, D. F.; McEwen, C. N.; Upham, R. A. Anal. Chem. 1972, $44,1292$.

11. Lin, Y. Y.; Smith, L. L. Biomed. Mass Spectrom. 1979, 6, 15. 
12. Buchanan, M. V. Anal. Chem. 1982, 54, 570-574.

13. Buchanan, M. V. Anal. Clem. 1984, 56, 546-549.

14. Liu, R. H.; Low, I. A.; Smith, F. P.; Piotrowski, E. G.; Hsu, A. F. Org. Mass Spectrom. 1985, 20, 511-514.

15. Verma, S.; Pomerantz, S. C.; Kethi S. K.; McCloskey, J. A. Anal. Chem. 1986, 58, 2898-2902.

16. Siegel, M. M. Anal. Chem. 1988, 60, 2090-2095.

17. Siegel, M. M. Proceedings of the 4th Texas Symposium, College Station, TX, 1988; pp. 179-186.

18. Dagan, S.; Amirav, A. Proceedings of the 43rd ASMS Meeting, Atlanta, GA, 1995; p 108

19. Wang, D.; Evans, R. J. Proceedings of the 4.3rd ASMS Meeting; Atlanta, GA, 1995; p 109.

20. Dagan, S.; Amirav, A. Int. J. Mass Spectrom. Ion Processes 1994, $133,187-210$
21. Brink, G. O. Rev. Sci. Instrum. 1966, 37, 857-860 and 1626.

22. Dagan, S.; Amirav, A. J. Am. Soc. Mass Spectrom. 1993, 4 869-873.

23. Dagan, S.; Amirav, A.; Fujii, T. Int. 1. Mass Spectrom. Ion Processes 1995, 151, 159165

24. Dagan, S.; Amirav, A. I. Am. Soc. Mass Spectrom. 1996, in press.

25. Cooks, R. G.; Ast, T.; Mabud, Md. A. Int. J. Mass Spectrom. Ion Processes 1990, 100, 209-265.

26. Fradeep, T.; Miller, S. A.; Cooks, R. G. I. Am. Soc. Mass Spectrom. 1993, 4, 769-773.

27. Kane, T. E.; Somogyi, A.; Wysocki, V. H. Org. Mass Spectrom. 1993, 28, 1665-1673.

28. McCormack, A. L.; Somogyi, A.; Dongre, A. R.; Wysocki, V.H. Anal. Chem. 1993, 65, 2859-2872. 